\title{
How did generalized solutions arise in solid mechanics?
}

\author{
L. P. LEBEDEV and R. O. GROSSI, Programa de Matemática \\ Aplicada de Salta, Facultad de Ingeniería, Universidad Nacional de \\ Salta, Avenida Bolivia 5150, 4400 Salta, República Argentina. \\ 〈promas@ciunsa.edu.ar〉〈grossiro@unsa.edu.ar〉
}

\section{Received 23rd November 1999}

Revised 5th March 2001

In recent decades, engineers and physicists have shown an increasing interest in functional analysis and its applications. As many of these practitioners lack special training in mathematics, they sometimes run into trouble when trying to use the tools of this powerful branch of knowledge. Our purpose is to outline the connection between the traditional ideas of mechanics and the newer mathematical concepts of generalized solution and distribution.

Key words: generalized solutions, functional analysis, Sobolev spaces, distribution

\section{INTRODUCTION}

Many mechanical engineers would like to understand the modern mathematics underlying mechanics; unfortunately, they are often intimidated by such terminology as 'generalized derivative' and 'Sobolev space'. An engineer whose main interest lies in applications faces great difficulty in bringing the tools of functional analysis to bear on his or her problems. Although functional analysis was developed by mathematicians with applications in mind, it was couched in language that physicists and engineers, the supposed beneficiaries of the theory, are largely unable to follow. This has unfortunately led to the suspicion that this powerful branch of mathematics was invented by mathematicians as a toy for other mathematicians. Nothing could be further from the truth: functional analysis was truly developed for the benefit of those who specialize in applications. Much of the subject area consists of extensions of the methods which engineers and physicists were already using (and, in fact, had invented themselves). This generalization of the existing tools merely served to place engineering mathematics on a firm foundation.

With the ever increasing availability and usage of 'black box' computer codes, it is now more important than ever for engineers to understand the principles which lie at the heart of modern numerical methods. The powerful and popular finite element method (FEM), for example, is based partly on the notion of generalized solution. We would therefore like to assist the newcomer in making the transition between the traditional ideas of mechanics and some newer ideas from applied mathematics. Many engineers will surely want to possess 
these fundamental ideas, even if they are not motivated to pursue functional analysis to any great extent.

It is known that Newton at first held back some of his achievements by publishing them in an abbreviated form. At times he described his own ideas quite simply, with words amounting to little more than 'It is useful to solve problems'. In the spirit of the great man we shall present a very important idea through a brief consideration of two old problems. We would like to explain where, how and why generalized solutions appeared in mechanics. Consider a plane membrane with clamped edge and under load $q(x, y)$. From mechanics it is known that the membrane is in equilibrium if and only if the energy integral

$$
\iint_{S}\left(\left(\frac{\partial u}{\partial x}\right)^{2}+\left(\frac{\partial u}{\partial y}\right)^{2}-2 q u\right) \mathrm{d} x \mathrm{~d} y
$$

attains its minimum over the admissible set of transverse displacements $u(x, y)$ of the membrane, which occupies domain $S$. The calculus of variations asserts that the minimum is attained if the equation (the first variation of the energy functional)

$$
\iint_{S}\left(\frac{\partial u}{\partial x} \frac{\partial \delta u}{\partial x}+\frac{\partial u}{\partial y} \frac{\partial \delta u}{\partial y}-2 q \delta u\right) \mathrm{d} x \mathrm{~d} y=0
$$

holds for any smooth function $\delta u$ that satisfies

$$
\left.\delta u\right|_{\partial S}=0
$$

The tools of the calculus of variations then imply that $u$ satisfies Poisson's equation

$$
\Delta u=-q
$$

To formulate the problem we must add a boundary condition

$$
\left.u\right|_{\partial S}=a(s)
$$

In old textbooks on the equations of mathematical physics, the existence of a solution of the Dirichlet problem, equations (3)-(4), possessing continuous first and second derivatives in $\bar{S}$ (denoted $u \in C^{(2)}(\bar{S})$ ) is demonstrated. The method employed is potential theory. These same textbooks then demonstrate uniqueness of solution. This is done as follows. Assume there are two solutions $u_{1}$ and $u_{2}$ that satisfy equations (3) and (4). Denote $v=u_{2}-u_{1}$ and subtract the equation (1) for $u_{1}$ from the equation (1) for $u_{2}$. Equation (3) is a consequence of equation (1) but equation (1) can be obtained from equation (3). So for $v$, that is a solution of equation (3) with $q=0$, we can write immediately

$$
\iint_{S}\left(\frac{\partial v}{\partial x} \frac{\partial \delta u}{\partial x}+\frac{\partial v}{\partial y} \frac{\partial \delta u}{\partial y}\right) \mathrm{d} x \mathrm{~d} y=0
$$

Since $\left.v\right|_{\partial S}=0$, it is possible to put $\delta u=v$, hence

$$
\iiint_{S}\left(\left(\frac{\partial v}{\partial x}\right)^{2}+\left(\frac{\partial v}{\partial y}\right)^{2}\right) \mathrm{d} x \mathrm{~d} y=0
$$

It follows that $\partial v / \partial x=\partial v / \partial y=0$ in $S$; in view of the boundary condition for $v$, we get $v=0$. 
In some problems the load $q$ is such that there is no solution having continuous second derivatives in $\bar{S}$. For example, suppose $q$ has a step increase at a line dividing the domain into two adjacent parts. A discontinuity in $q$ implies, by equation (3), a corresponding discontinuity in $\Delta u$, hence at least one of the second derivatives must have a break at the line. Those who restrict themselves to classical mathematical physics have difficulty proving the existence of such solutions. They often try to circumvent this difficulty by considering two coupled problems and then studying the behaviour of the respective solutions near the dividing line. However, the use of an FEM code can easily give a good approximation to the solution which agrees with the experiment, and which does not necessitate splitting the domain. To formulate the FEM equations one can use equation (1) directly, not turning to equation (3). This brings us to the idea of the direct exploitation of equation (1) to formulate the problem of equilibrium of the membrane, and also leads to the idea of a generalized solution. So a generalized solution, meeting boundary conditions, equation (4), must satisfy equation (1) for any $\delta u$ satisfying equation (2). Such a solution is called a weak solution. It is seen that equation (1) only requires $u$ to have first derivatives. Moreover, it is not necessary for these to be continuous; it is quite enough if one can calculate all the integrals in equation (1). The proof of uniqueness of a classical solution can be repeated without modification for the weak solution, hence the weak solution is unique.

This naive formulation of the weak solution is enough to enjoy the fruits of the FEM. However, those who wish to obtain rigorous results regarding convergence of FEM approximations will run into trouble using the usual calculus notions of derivative and Riemann integral. The reader is undoubtedly aware of the difficulties encountered in elementary calculus when one tries to pass limits through the integration symbol. To get rigorous results in this area more easily, mathematicians invented the Lebesgue integral and the generalized derivative. We shall not offer an explanation of the Lebesgue integral; an elementary but not too simple introduction can be found in [1]. It should merely be noted that in case of a function $f(x)$ continuous on $\bar{S}$, the Lebesgue integral of $f(x)$ over $S$ is equal to the corresponding Riemann integral.

We now demonstrate how mathematicians introduce generalized derivatives of functions which may lack usual derivatives. Let a continuous function $u(x, y)$ have a continuous derivative with respect to $x$ at each point of a bounded set $\bar{S}$. Take a function $\varphi(x, y)$ that (a) is infinitely differentiable, and (b) equals zero in some neighbourhood of the boundary $\partial S$ (such a function is said to be finite and infinitely differentiable). The integration by parts formula

$$
\iint_{S} u \frac{\partial \varphi}{\partial x} \mathrm{~d} x \mathrm{~d} y=-\iint_{S} \frac{\partial u}{\partial x} \varphi \mathrm{d} x \mathrm{~d} y
$$

is valid. We momentarily forget equation (5) and suppose that for a given $u$ the equality

$$
\iint_{S} u \frac{\partial \varphi}{\partial x} \mathrm{~d} x \mathrm{~d} y=-\iint_{S} v \varphi \mathrm{d} x \mathrm{~d} y
$$

holds for any finite and infinitely differentiable function $\varphi(x, y)$. It can be shown that any two functions $v_{1}$ and $v_{2}$ for which equation (6) holds must coincide almost everywhere (i.e., they can differ only on a set whose Lebesgue measure is zero). Comparing equations (5) and (6), we see that in the case of a differentiable function $u(x, y)$, equation (6) defines its derivative $v=\partial u / \partial x$. If $u(x, y)$ has no continuous derivative but equation (6) holds for any finite and infinitely differentiable function $u$, then it is natural to call $v$ the generalized 
derivative and denote it by $\partial u / \partial x$. It is seen that the necessary restriction on $u(x, y)$ to possess a generalized derivative $\partial u / \partial x$ is that it be integrable. Sobolev [2] was the first who, using Lebesgue integration, introduced generalized derivatives into the study of boundary value problems. Generalized derivatives possess some properties that usual derivatives do not. For example, if a function $f(x)$ does not have an ordinary derivative, then the function $w(x, y)=f(x)+f(y)$ has the generalized derivative $\partial^{2} w / \partial x \partial y$ that is equal to zero.

It is seen that equation (1), defining the weak solution, and equation (6), defining the generalized derivative, have quite similar origins. But the weak solution was introduced via the minimal principle. Mathematicians prefer to introduce generalized solutions as the generalized derivative was introduced above. Let us demonstrate how this is done for the problem, equations (3)-(4). Multiply both sides of equation (3) byy a finite infinitely differentiable function $\varphi(x, y)$ and integrate the result over $S$. We get

$$
\iint_{S} \Delta u \varphi \mathrm{d} x \mathrm{~d} y=-\iint_{S} q \varphi \mathrm{d} x \mathrm{~d} y
$$

This equation can serve as a basis for the introduction of a generalized solution to equation (3)-(4). In this definition one need require only integrability of the second derivatives of the solution $u(x, y)$. The integral in the left hand side is

$$
\iint_{S} \Delta u \varphi \mathrm{d} x \mathrm{~d} y=\iint_{S}\left(\frac{\partial^{2} u}{\partial x^{2}} \varphi+\frac{\partial^{2} u}{\partial y^{2}} \varphi\right) \mathrm{d} x \mathrm{~d} y
$$

Let us integrate by parts in the first addendum of the integrand of the right side

$$
\iint_{S} \frac{\partial^{2} u}{\partial x^{2}} \varphi \mathrm{d} x \mathrm{~d} y=-\iint_{S} \frac{\partial u}{\partial x} \frac{\partial \varphi}{\partial x} \mathrm{~d} x \mathrm{~d} y+\oint_{\partial S} \frac{\partial u}{\partial x} \varphi n_{x} \mathrm{~d} s=-\iint_{S} \frac{\partial u}{\partial x} \frac{\partial \varphi}{\partial x} \mathrm{~d} x \mathrm{~d} y
$$

where $n_{x}$ is the $x$-component of the outward normal to the boundary and the contour integral vanishes because $\varphi$ is equal to zero on the boundary $\partial S$. Similarly,

$$
\iint_{S} \frac{\partial^{2} u}{\partial x^{2}} \varphi \mathrm{d} x \mathrm{~d} y=-\iint_{S} \frac{\partial u}{\partial y} \frac{\partial \varphi}{\partial y} \mathrm{~d} x \mathrm{~d} y+\oint_{\partial S} \frac{\partial u}{\partial y} \varphi n_{y} \mathrm{~d} s=-\iint_{S} \frac{\partial u}{\partial y} \frac{\partial \varphi}{\partial y} \mathrm{~d} x \mathrm{~d} y
$$

Combining these, from equation (7) we derive the equation

$$
-\iint_{S}\left(\frac{\partial u}{\partial x} \frac{\partial \varphi}{\partial x}+\frac{\partial u}{\partial y} \frac{\partial \varphi}{\partial y}\right) \mathrm{d} x \mathrm{~d} y=-\iint_{S} q \varphi \mathrm{d} x \mathrm{~d} y
$$

Aside from notation we have obtained equation (1). The only difference is that in equation (1) we did not require $\delta u$ to be finite and infinitely differentiable. This requirement of finiteness and infinite differentiability for $\varphi(x, y)$ can cause additional trouble if we develop a modification of the FEM, since in the FEM the basis functions are normally not smooth.

Let us proceed, integrating by parts in equation (8)

$$
-\iint_{S} \frac{\partial u}{\partial x} \frac{\partial \varphi}{\partial x} \mathrm{~d} x \mathrm{~d} y=\iint_{S} \mathrm{u} \frac{\partial^{2} \varphi}{\partial x^{2}} \mathrm{~d} x \mathrm{~d} y-\oint_{\partial S} \frac{\partial u}{\partial x} \varphi n_{x} \mathrm{~d} s=\iint_{S} u \frac{\partial^{2} \varphi}{\partial x^{2}} \mathrm{~d} x \mathrm{~d} y
$$

and similarly 


$$
-\iint_{S} \frac{\partial u}{\partial y} \frac{\partial \varphi}{\partial y} \mathrm{~d} x \mathrm{~d} y=\iint_{S} \mathrm{u} \frac{\partial^{2} \varphi}{\partial y^{2}} \mathrm{~d} x \mathrm{~d} y-\oint_{\partial S} \frac{\partial u}{\partial y} \varphi n_{y} \mathrm{~d} s=\iint_{S} u \frac{\partial^{2} \varphi}{\partial y^{2}} \mathrm{~d} x \mathrm{~d} y
$$

Combining these, we get

$$
\iint_{S} u \Delta \varphi \mathrm{d} x \mathrm{~d} y=-\iint_{S} q \varphi \mathrm{d} x \mathrm{~d} y
$$

This requires of $u(x, y)$ nothing except integrability. Mathematicians also study this and even more general formulations for generalized solutions. These are useful because they reveal much about the nature of Poisson's equation. But in the generalization when a solution is only integrable the problem can have other solutions.

In mechanics it is reasonable to stop generalizing when such problems have no more than one solution, hence for the problem under consideration it is reasonable to stop at either definition, equations (8) or (1). But there is another, deeper reason for the generalization, equation (8): its solutions correspond to the states of the membrane with finite energy. Unlike mathematicians we prefer to use trial functions $\varphi(x, y)$, in the definition of the generalized solution, which are of the same nature as the solution itself. A reader interested in rigour should refer to those books that take the time to discuss which sets of functions have generalized derivatives that are square integrable. Many of these sources will lead the reader to the theory of Sobolev spaces. These are spaces of functions having generalized derivatives up to the order $l$ that are integrable with some degree $p \geqslant 1$ over the domain $\Omega$, and are denoted by $W_{p}^{(l)}(\Omega)$ or $W^{l, p}(\Omega)$. For additional details of the theory of these important spaces see reference [3]. Students can find an excellent exposition in reference [4].

\section{DISTRIBUTIONS}

We now present another simple problem that can be solved by a junior-level engineering student, and that will again demonstrate how the generalization of a problem set-up can bring us to generalized solutions. Consider a cantilever beam under a distributed load $q$, a point force $F$, and a torque $M$ (Fig. 1).

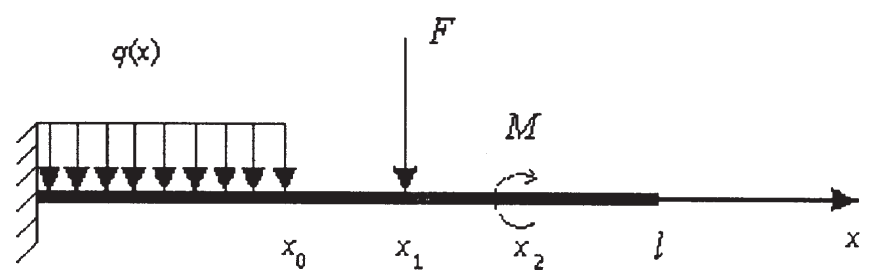

Fig. 1.

The equilibrium of a beam under a transverse load $q(x)$ is governed by

$$
\operatorname{EIu}^{(4)}(x)=q(x)
$$

The point force $F$ and the point torque $M$ applied to the beam do not allow us to write out a unique equation for the bending problem. To solve this problem, three simple coupled problems are normally considered: the first on the interval $\left(0, x_{1}\right)$, governed by equation 
(10); the second on the interval $\left(x_{1}, x_{2}\right)$; the third for $x>x_{2}$. Resolving every equation (often done graphically) and then solving a system of equations for coupling of the problems, we can get the solution. However, it is possible to write out the unique equation for the problem using the principle of virtual work

$$
\begin{aligned}
& \int_{0}^{l} E I u^{\prime \prime} \delta u^{\prime \prime} \mathrm{d} x=\int_{0}^{l} q \delta u \mathrm{~d} x+Q \delta u\left(x_{1}\right)+M \frac{\partial \delta u\left(x_{2}\right)}{\partial x} \\
& u(0)=0, \quad u^{\prime}(0)=0
\end{aligned}
$$

We consider $q$ to be zero on the interval $\left[x_{0}, l\right]$. From these it is possible to derive equation (10) and, moreover,, the natural boundary conditions at the free edge of the beam. Equation (11) can be used for the formulation of the FEM equations directly. We can use it to introduce the notion of generalized solution for the problem under consideration as follows. Let us introduce the class $E$ of functions having the first two generalized derivatives square integrable and satisfying the boundary conditions, equation (12). We call $u \in E$ the generalized solution of the problem under consideration if $u$ satisfies equation (11) for any $\delta u \in E$. It can be shown that this statement defines $u$ uniquely and that it exists if $q$ is integrable. So the concept is well defined and, moreover, the usual solution of the problem is a generalized solution, so we have no additional trouble if we find it in the traditional manner.

However, equation (11) can easily be generalized for other problems where the beam has a variable cross section (it is enough to simply include the dependence of $I$ on $x$ ), and it is possible to consider a case where the end of the beam is elastically supported and elastically restricted against rotation. In this case

$$
\begin{aligned}
\int_{0}^{l} E I u^{\prime \prime} \delta u^{\prime \prime} \mathrm{d} x= & \int_{0}^{l} q \delta u \mathrm{~d} x+Q \delta u\left(x_{1}\right)+M \frac{\mathrm{d} \delta u\left(x_{2}\right)}{\mathrm{d} x} \\
& +k u(l) \delta u(l)+r \frac{\mathrm{d} u(l)}{\mathrm{d} x} \frac{\mathrm{d} \delta u(l)}{\mathrm{d} x}
\end{aligned}
$$

where $k$ and $r$ are the rigidities of the elastic supports. To formulate the generalized set-up of the new problem, it is enough to take the above definition and replace equation (11) by equation (13).

In a similar manner, we can formulate problems for finding eigenfrequencies of a beam with elastically supported parts and points. We can also formulate linear and nonlinear problems for plates, shells, etc. It is possible to consider non-elastic bodies, say, in the framework of viscoelasticity or plasticity. Such treatments give rigorous justification for applying the FEM. The particular mode of convergence exhibited by the FEM can then be understood. We shall not pursue this topic here, since it requires tools of functional analysis not assumed in this discussion.

Let us touch on another question; whether it is possible to write something like the unique classical equation for the problem (11)-(12). The answer is yes if we employ generalized derivatives again, but it will involve the derivative of a distribution, a more general object. We begin with the notion of the $\delta$-function that is used by any physicist.

Take the step function

$$
h(x)=\left\{\begin{array}{lll}
1 & \text { if } & x \geqslant 0 \\
0 & \text { if } & x<0
\end{array}\right.
$$

Let $\varphi(x)$ be finite on $\mathbb{R}=(-\infty,+\infty)$ and infinitely differentiable, and calculate 


$$
\int_{-\infty}^{\infty} h(x) \frac{\mathrm{d} \varphi(x)}{\mathrm{d} x} \mathrm{~d} x=\int_{0}^{\infty} 1 \bullet \frac{\mathrm{d} \varphi(x)}{\mathrm{d} x} \mathrm{~d} x=\left.\varphi(x)\right|_{0} ^{\infty}=-\varphi(0)
$$

Paul Dirac proposed to write out the right hand side of equation (14) as

$$
-\varphi(0)=-\int_{-\infty}^{\infty} \delta(x) \varphi(x) \mathrm{d} x
$$

Of course, no such function $\delta(x)$ satisfies this equality for any finite and infinitely differentiable function $\varphi(x)$. But Dirac introduced it, and several generations of physicists used it with the explanation that $\delta(x)$ acts somewhat like a function vanishing everywhere except at $x=0$ where it equals such an infinity that equation (15) holds. Later, mathematicians learned how to introduce objects like $\delta$-functions. They called these objects distributions, which are not functions but functionals of some spaces. The theory of distributions was introduced by L. Schwartz, reference [5]. For additional details see references [6] and [7].

A nice aspect of this theory is that one can differentiate all such distributions infinitely many times, and the definition of a distribution is quite similar to the one we used to introduce the generalized derivative. Substituting $-\varphi(0)$ from equation (15) into equation (14) we get

$$
\int_{-\infty}^{\infty} h(x) \frac{\mathrm{d} \varphi(x)}{\mathrm{d} x} \mathrm{~d} x=-\int_{-\infty}^{\infty} \delta(x) \varphi(x) \mathrm{d} x
$$

If $h(x)$ were a continuously differentiable function, the formula of integration by parts would be

$$
\int_{-\infty}^{\infty} h(x) \frac{\mathrm{d} \varphi(x)}{\mathrm{d} x} \mathrm{~d} x=-\int_{-\infty}^{\infty} \frac{\mathrm{d} h(x)}{\mathrm{d} x} \varphi(x) \mathrm{d} x
$$

and thus, comparing this with equation (16) we can formally conclude that

$$
\delta(x)=\frac{\mathrm{d} h(x)}{\mathrm{d} x}
$$

In a similar way, we can formally introduce $\mathrm{d} \delta(x) / \mathrm{d} x$ that becomes the second derivative $\mathrm{d}^{2} h / \mathrm{d} x^{2}$, etc.

Using these expressions for the $\delta$-function, we can write out the equation of the problem (11) formally as the unique expression

$$
\frac{\mathrm{d}^{2}}{\mathrm{~d} x^{2}}\left(E I \frac{\mathrm{d}^{2} u(x)}{\mathrm{d} x^{2}}\right)=q(x)+Q \delta\left(x-x_{1}\right)+M \frac{\mathrm{d}}{\mathrm{d} x} \delta\left(x-x_{2}\right)
$$

This holds in the interval $(0, l)$, and must be supplemented by conditions (equation (12)) and the natural boundary conditions at $x=l$.

So we see that some new ideas from mathematics have returned us to the old ideas of mechanics once again.

\section{REFERENCES}

[1] Lebedev, L. P., Vorovich, I. I. and Gladwell, G. M., Functional Analysis: Applications in Mechanics and Inverse Problems, Kluwer Academic Publishers, (1996). 
[2] Sobolev, S. L., Applications of Functional Analysis in Mathematical Physics, American Mathematical Society, Providence, Rhode Island (1963).

[3] Adams, R. A., Sobolev Spaces, Academic Press, NY (1975).

[4] Rektorys, K., Variational Methods in Mathematics, Science and Engineering, D. Reidel Co., Dordrecht, Holland (1980).

[5] Schwartz, L., Theorie des Distributions, Hermann, Paris (1951).

[6] Necas, J., Les méthodes directes en théorie des équations elliptiques, Masson, Paris (1976).

[7] Blanchard, P. and Bruning, E., Variational Methods in Mathematical Physics, Springer, Berlin (1992). 